\title{
Poesia come provocazione: itinerari espressivi di Antonio Porta*
}

Per i poeti della neo-avanguardia ogni ermeneutica della realtà poetica inizia da un'ermeneutica del linguaggio. Alfredo Giuliani, nell'introduzione all'antologia I Novissimi, precisa tale concetto contrario alle implicazioni delle poetiche novecentesche pre-belliche e post-belliche, di crepuscolarismo, ermetismo, neo-sperimentalismo - nei seguenti termini:

Perché ci siamo tanto preoccupati del lessico, della sintassi, del metro e via dicendo? Perché se conveniamo che, in quanto "contemporanea," la poesia agisce direttamente sulla vitalità del lettore, allora ciò che conta in primo luogo è la sua efficacia linguistica. ${ }^{1}$

Nelle creazioni poetiche proposte dalla neo-avanguardia "non saranno piú - come afferma Balestrini, altro esponente del gruppo i Novissimi - il pensiero e l'emozione a venire trasmessi per mezzo del linguaggio, ma sarà il linguaggio stesso a generare un significato nuovo e irripetibile." 2 Tutto ciò acquista un valore marcatamente polemico nei confronti del codice contemporaneo, contaminato dal livellamento dei mezzi di diffusione, dalla mercificazione consumistica dei prodotti estetici, dalle mistificazioni dei meccanismi pubblicitari, che hanno operato una riduzione semantica del segno, e quindi un intorpidimento dello spirito critico nei confronti delle strutture del reale manovrate da tale segno linguistico.

Ai Novissimi non rimane pertanto altra scelta che estraniare il linguaggio "dalle sue proprietà semantiche, lacerandone il tessuto sintattico, scomponendone l'armonia, e ricostruendolo in ordini provvisori violentemente sincronici"; "dedicarsi al montaggio asemantico dei segni"3 e pervenire ad una poesia "schizomorfa," ad un progetto poetico inteso come critica della realtà, come "mimesi critica" di "rispecchiamento e contestazione." 4

Attraverso l'esercizio di un ludico cinismo, il poeta compie un atto di intenzionale straniamento nei confronti della "visione nor- 
male, e normalmente sancita e riconosciuta, della realtà contemporanea" ${ }^{\prime 5}$ e ponendosi in una prospettiva radicalmente altra rispetto ad essa, non può che dedicarsi alla comunicazione della negazione della comunicazione esistente.

Il messaggio poetico, insomma, può prodursi solo attraverso il rifiuto del codice. Il codice si rifiuta in quanto si rifiutano i suoi referenti, i significati ideologici che la lingua veicola. ${ }^{6}$ La poesia pertanto, come si diceva, diventa ricerca verbale, esplorazione del linguaggio. ". . . prima di guardare alla astratta ideologia, all'intenzione culturale," - scrive Giuliani - "noi guardiamo alla semantica concreta della poesia, senza dimenticarci che essa è dopotutto un'arte, una vis mitologica: per gli ideologi, al contrario, la poesia è soltanto un pretesto e come tale disposta ad essere Circe o la scrofa, a seconda dello scopo cui deve servire."

Nella sua funzione catalizzatrice di innovamenti, la poesia neoavanguardistica si oppone logicamente anche alle ideologie precostituite che strumentalizzano il prodotto estetico assoggettandolo alle categorie del bello, del piacevole, del godibile, di un'armonica corrispondenza fra segno e oggetto: no all'edonismo, alle suggestioni di romantici o crepuscolari sfoghi intimistici, a definitorie ipotesi di salvezza. ${ }^{8}$

Il linguaggio, eversivamente sottratto ad impalcature ordinatrici tradizionali, non agisce, comunque, solo come promotore di scontri e svecchiamento di dogmi fossilizzati, ma si impone anche come mezzo di ricognizione di determinate realtà contemporanee, dominate dall'alienazione e reificazione. In altri termini, viene smontata l'illusione di poter contestare la situazione odierna assumendo un linguaggio che si mantenga immune dalla crisi contestata.

Tale direttiva, infatti, alienerebbe arbitrariamente il linguaggio dalla problematica descritta, come rileva Eco nella sua Opera aperta: se infatti uno scrittore, per comunicarci la crisi di rapporti umani distorti e sconnessi, usa un linguaggio ordinato, si rende colpevole di un atto mistificatorio: "Quest'ordine è ovviamente fittizio, è l'ordine delle strutture narrative che esprimevano un universo ordinato, quest'ordine costituisce una forma di giudizio pronunciato nei termini di un linguaggio estraneo alla situazione."9

I Novissimi contestano ogni forma di neo-crepuscolarismo piangevole od ogni tentativo di sfuggire la "realtà matrigna" mediante "schemi di un razionalismo parentetico e velleitario."10 All'autobiografismo lacrimevole, al pianto sull'infelicità storica essi oppongono la lucida consapevolezza dell'impotenza della poesia di fronte alla realtà in crisi. Si respinge ogni illusione di un'operazione poetica capace di rompere o risolvere crisi. Spetterà solo alla 
prassi rovesciare la realtà e farsi portatrice di una carica liberatoria atta a capovolgere le condizioni storiche del presente.

Posto di fronte ad un'età schizofrenica il poeta può solo compiere un "actus tragicus,"11 patire fino in fondo la realtà al negativo, affondare nella "livida Palus"12 e rivivere, trascrivere sulla pagina l'"oggettivo esaurimento storico."13

Non una "rimozione" dunque della realtà in crisi ma un atto di tragica consapevolezza, consapevolezza come unica possibilità di denuncia e di lotta. Chiamato ad affrontare una "società/materasso, gommapiuma, carta/assorbente," vole" che "rifiuta l'urto" per invischiare, assorbire od incorporare i colpi, il poeta è consapevole che "una diretta violenza è del tutto inefficace in un'età tappezzata di viscide sabbie mobili." ${ }^{15}$ Egli sceglierà una poesia come opposizione, come negazione e romperà ogni legame con la poesia intesa come seduzione: al corteggiamento opporrà il terrorismo, al lirismo la sadica frantumazione del materiale verbale.

Nella sua carica eversiva e demolitrice, la poesia dei Novissimi sconvolgendo le attese formali, retoriche, "porta il destinatario a ridimensionare le sue attese ideologiche ... e mette in crisi le ideologie e i codici che le veicolano."16 Di conseguenza assistiamo alla tendenza di bloccare la significazione e il significante diventa il nuovo significato. In altre parole, la strutturazione formale si traduce in neocontenutismo ed il linguaggio diventa non solo manifestazione del reale ma oggetto di se stesso. A tale proposito Fausto Curi cosí scrive:

Sollevato dalla sua funzione di medium, il soggetto dell'operazione poetica diventa il linguaggio. E poiché il linguaggio non può, evidentemente, dire che se stesso, esso diventa all'istante l'oggetto della propria operazione. ${ }^{17}$

Nell'ambito di tali prospettive si colloca l'opera poetica di Antonio Porta, che rifiuta il concetto del testo poetico inteso come seduzione, e proponendosi come costante campo d'indagine la strutturazione fisica del materiale verbale e dello spazio sintattico in cui esso si muove, demistifica i significati, tenta la conquista di nuovi universi semantico-formali e costruisce testi aperti e provocatori, atti ad instaurare un rapporto inquietante e positivamente ambiguo fra testo e destinatario.

Nel rifiuto di ideologie univoche e dogmatiche, il poeta scarta ogni mediazione soggetto-oggetto, basata su una coscienza che intervenga a razionalizzare e catalogare i dati della realtà, e tramite la riduzione dell'io, la messa in parentesi della propria soggettività, vuole pervenire ad un'autonomia del piano dell'e- 
spressione. Infatti, in una dichiarazione di poetica Porta scrive:

Il carattere non-metaforico della nuova poesia . . . vuol significare che il contesto poetico non è metafora del mondo $\mathrm{e}$, in definitiva, nemmeno il suo "correlativo," ma mondo in sé. ${ }^{18}$

Il segno poetico non va, pertanto, strumentalizzato dall'incalzare di valori già fissati prima dell'operazione poetica: "l'ideologia ante poiesim - sostiene l'autore - quella che non nasce, cioè, dal fare e dal risultato poetici, non può trovarci in alcun modo consenzienti. ${ }^{19}$ Va notato, comunque, che pur rifiutando una scrittura autoritaria che imponga l'informazione come verità irrevocabile ed ultima, l'operazione poetica portiana comunica anch'essa una visione del reale, un'interpretazione del mondo da parte dell'artista: e non si tratta - badiamo bene - di una contraddizione, ma di una necessaria conseguenza dell'uso del linguaggio, che è sempre un modo di interpretare la realtà, un'ideologia (come sostiene Sanguineti nel suo Ideologia e linguaggio). ${ }^{20}$ In altre parole, una scrittura "aideologica," "neutrale," è inevitabilmente illusoria, in quanto essa non è mai innocente, è sempre uno strumento condizionato da un'ideologia. Ciò che separa Porta dalla tradizione è il fatto che questa visione del reale non guida a priori la scelta e l'organizzazione del materiale poetico, ma è risultato stesso dell'operazione creativa, e preclude l'ancoramento statico a sistemi di valori definitivi, come dimostra il continuo rivoluzionamento di prospettive dell'intera produzione portiana, che non esita a contraddirsi o contestare le sue stesse fasi precedenti nella beffarda e stimolante smentita di ottimismi gnoseologici lungimiranti. "Il mondo poetico" quindi, nelle parole di Porta, "pur mantenendo la sua autonomia originaria, una volta raggiunta l'organicità senza la quale non potrebbe esistere, diventa anche conoscenza e riflesso della storia, in senso non soltanto 'rappresentativo,' ma a volta a volta, prospettico, utopico, ipotetico, nei modi in cui il poeta proponendo all'attenzione dei lettori il meccanismo che ha costruito si rivela disposto a determinate ipotesi di vita." 21

Se la esaminiamo sotto un profilo filosofico, la genesi della poesia portiana "in re" gravita intorno ad uno dei fondamenti della fenomenologia, ossia il fatto che l'esistenza del mondo viene riconosciuta come precedente al sorgere della filosofia, e indipendente da essa: il reale va affrontato tramite un ritorno alle cose, al mondo della percezione, in cui gli oggetti sono seguiti nel loro temporale esplicarsi. Inoltre, dal momento che il nostro Dasein ci condanna ad affrontare esperienze ben definite e limitate, non è possibile arrivare ad uno stadio mentale conclusivo che abbracci 
tutto il pensiero possibile: pertanto la filosofia, la nostra Weltanschauung, è sempre sul punto di rinascere, di ricostruire le sue premesse sul momento, senza essere contagiata da verità scontate o globali: ". . . non il tuffo ingenuo inutilmente temuto, nel mare dell'oggettività - avverte Porta a questo riguardo - ma l'articolarsi del conoscere, nel nostro ora.

La concentrazione sul linguaggio inteso come autonomo materiale da costruzione potrebbe rappresentare come pericolo immediato la caduta nel puro formalismo. Porta si manifesta, ben consapevole della necessità di "abbandonare il puro gioco verbale, elegante finché si vuole, e le strutture gratuite." ${ }^{23}$ Dietro la persistente disgregazione tecnica del materiale linguistico, infatti, si manifesta la costante concentrazione del poeta sul problema del vero: "Qualcosa si vuol trovare, alla fine - sostiene Porta - le cose che manovriamo o ci manovrano, i fatti che determiniamo o che ci determinano, sono certamente in relazione con la verità: proprio per avvicinarla ci serviamo del vero, cercandolo negli oggetti e negli eventi." 24 È proprio questa tensione maieutica che, pur se refrattaria ad approdi definitivi, riscatta le operazioni contestatarie sul linguaggio dai limiti dello sperimentalismo formale, e le nobilita, al contrario, riflettendo in loro la tragicità jaspersiana di ricerche inappagate e sempre sul punto di rinascere. ${ }^{25}$

Per fornire un'esemplificazione pratica della poetica portiana sin qui delineata, abbiamo selezionato tre testi provenienti da fasi ben distinte della produzione dell'autore, che esamineremo individualmente premettendo che le verifiche testuali che seguiranno inquadreranno solo sommariamente gl'itinerari espressivi del poeta e gli eterogenei slanci contestatari in essi reperibili.

\section{Campione $n .1$}

che la libertà è prima di tutto un dovere che la libertà sta nell'aderire alla legge che il destino dell'uomo è segnato fin dalla nascita che l'amore è destinato a diminuire col tempo che si vede dalla faccia che uno è un porco che bisogna dire di sí il piú tardi possibile che bisogna dire di no per farsi rispettare che vivere insieme ai cinesi è impensabile che è morto per difendere la sua innocenza che è morto perché dava via il culo perché non lo voleva dare che $\mathrm{i}$ veggenti effettivamente indovinano che la verginità è un dono 
che una volta messi su quella strada non si torna piú indietro che tanto vale dire sí e perché no che dobbiamo chiederci perché sí che le cerimonie d'investitura non sono piú splendide che i cardinali non divertono piú che le bombe possono metterle solo i figli dei ricchi che gli studenti non pensano a fare l'amore che gli studenti occupano le università solo per accoppiarsi che le lettere d'amore di Marx non esistono che le lettere d'amore di Rosa Lux ci fanno bene sperare che i coiti a gruppi sono passati di moda che $i$ vescovi sono diventati tolleranti che la polizia sa chi è il capo ma non può dirlo l'omosessualità è o non è una vergogna non c'è altro da fare che attendere ${ }^{26}$

Nel primo campione da noi fornito ogni singolo verso ci rimanda al titolo, Quello che tutti pensano, e quest'ultimo assume una funzione unificatrice per tutte le dieci sezioni che compongono la prima parte della raccolta Metropolis. La struttura generale impiegata dal poeta è assai semplice ed elementare. Si tratta di una trasposizione scritturale in forma di catalogo di svariati luoghi comuni, un elenco di topoi definitori che riproducono il comportamento linguistico e i vari contenuti con cui abbiamo quotidianamente a che fare. Segno paradigmatico delle impalcature linguistiche della cultura contemporanea qui denunciata è la definizione, vale a dire il tratto distintivo di un linguaggio chiuso, anticritico ed antidialettico, pronto ad irrigidire e a banalizzare, che nella sua funzionale organizzazione diventa veicolo di falsificazione dell'informazione, assumendo quindi anche i connotati di un linguaggio autoritario e dogmatico. Quest'ultimo è un codice che, come rileva anche Marcuse, assorbe gli elementi trascendentali e negativi della ragione. ${ }^{27}$

La denuncia della falsa informazione come momento contestatario che investe il campo stesso della comunicazione poetica dunque autocritica della poesia - da un lato opera come messa in crisi della definizione-comunicazione, dall'altro risulterà come delucidazione e demistificazione dei procedimenti linguistici codificati, alienati ed alienanti. Il processo denunciatore e demistificatorio viene esplicato essenzialmente dall'organizzazione testuale: la selezione del materiale subisce una forte neutralizzazione semantica una volta che viene sottoposta ad una combinazione conflittuale, banale o estranea tout court. Insomma, gli atti combinatori saranno privati di un asse referenziale univoco oppure omogeneo, e la consequenziale conflittualità risulterà nello svuotamento semantico del materiale selezionato, nella demistifica- 
zione di esso e quindi nella rivelazione della falsità dell'informazione. $^{28}$

Tali procedimenti, inoltre, portano a galla il blocco e l'inerzia del pensiero causati dalla definizione, la manipolazione della coscienza la quale si aliena a un linguaggio reificato che preclude un contatto vero col mondo.

Il catalogo dei luoghi comuni, degli svariati cliché prescelti, è formato da sintagmi compiuti e indipendenti, combinati tra loro non per relazioni semantiche o tramite un campo denotativo ristretto, ma per la formalizzazione aforistica che hanno in comune. Tuttavia, le combinazioni sintagmatiche nella loro disposizione testuale non presentano un collocamento dettato da una cieca casualità, ma vengono inserite in un montaggio che rivela il calcolo demistificatorio degli accostamenti.

Il montaggio è effettuato principalmente per mezzo dei seguenti ricorsi: 1. definizioni che perdono la credibilità (e quindi rivelano la loro falsificazione) in quanto combinate con altre definizioni parallele dello stesso topos (es. vv.1-2); 2. sintagmi conflittuali di tipo affermazione-negazione (es. vv.6-7, 10-11, 22-23); 3. inserimento di sintagmi estranei o discordanti con il resto dei topoi (es. vv.5, 12, 15, 16).

L'apparentemente casuale combinatoria sintagmatica prevede l'accostamento di sintagmi collegati a disparati campi d'indagine. Nell'intera sezione della raccolta da cui sono stati tratti questi versi il poeta seleziona definizioni che vanno dalla politica al razzismo, dal sesso alla religione, dalla psicologia alla sociologia, dall'arte agli eventi piú banali.

Nei suoi svariati settori d'esperienza la nostra civiltà dei consumi ha un continuo bisogno di fruire di informazioni semplificate, schematizzate e dissociate dalla loro complessità originaria. Per fornire altri due brevissimi esempi, basti vedere come vengono ridotte le teorie di Freud ("che la sessualità è il motore di tutto") o di Marx ("che alla base ci sta il fatto economico che tutto determina") (sezione 5). Le generalizzazioni collettive riproducono una realtà che deforma e falsifica gl'intenti stimolanti che l'animavano. Insomma, anche il pensiero piú complesso viene ridotto a slogan superficiali meccanicamente inglobati da parte di inerti fruitori ed il linguaggio pertanto subisce la degradazione a oggetto di consumo.

Oltre alla tecnica del montaggio, cioè della combinazione di eterogenee definizioni, Porta adopera un procedimento di martellante reiterazione di formule sintattiche, inaugurate categoricamente dal "che" e centrate sulla perentorietà definitoria dell'indicativo, oltre che sulla comparsa frequente di verbi d'indirizzo 
conativo: tali accorgimenti evidenziano la standardizzazione seriale dei luoghi comuni elencati, e l'automatismo non riflessivo che ne favorisce la diffusione di massa.

La poesia, inoltre, immagazzinando nel suo interno i meccanismi falsificanti del linguaggio odierno, ironizza anche sulla sua stessa funzione contestataria, destinata ad essere fruita allo stesso modo degli slogan stereotipati e mercificabili che ospita eversivamente nelle sue strutture.

In altri termini, la contestazione alla mercificazione del linguaggio e della cultura, una volta inserita nel mercato allo scopo di comunicare se stessa, diventa immediatamente una componente del medesimo processo mercificante condannato, e la poesia, pertanto, rivela nella sua costruzione formale anche la sua consapevolezza di essere destinata ad una sconfitta "consumistica" sul piano del mercato, che ingloba facilmente, neutralizzandoli, gl'impulsi contestatari.

Campione n. 2

$\begin{array}{ll}\text { uomo/morte } & \text { uomo/terra } \\ \text { vita/morte } & \text { uomo/tomba } \\ \text { tomba/albero } & \text { polvere/tomba } \\ \text { cavallo/tomba } \\ \text { roccia/tomba } & \text { spade/vita } \\ \text { uomo/vita } & \text { lotte/vita } \\ \text { difese/vita } & \text { roccia/uomo } \\ \text { acqua/vita } & \text { uomo/scava } \\ \text { uomo/triangolo } & \text { batte/uomo } \\ \text { uomo/foglia } & \text { uomo/preda } \\ \text { tomba/uccello } & \text { preda/vita } \\ \text { preda/morte } & \text { uomo/morte } \\ \text { vivo/uomo } & \text { uomo/uccello } \\ \text { uomo/cavallo } & \text { tu/io } \\ \text { fuori/dentro } & \text { tutto/tu } \\ \text { l'altro/io } & \text { occhio/tomba } \\ \text { io/tutto } & \text { lingua/tomba } \\ \text { suono/tomba } & \text { lingua/pioggia } \\ \text { pioggia/tomba } & \text { romba/vita } \\ \text { lingua/vede } & \text { amo/morte } \\ \text { cerchio/morte } & \text { polvere/uccello } \\ \text { vita/amo } & \text { preme/terra } \\ \text { vola/morte } & \text { ardo/morte } \\ \text { striscio/vita } & \text { chiamo/tomba } \\ \text { sudo/vita } & \text { tetto/morte } \\ \text { tomba/vita } & \text { buca/vola } \\ \text { buca/morte } & \text { tengo/vita } \\ \text { stringo/tomba } & \text { tomba/senza } \\ \text { buca/vuoto } & \end{array}$


Il testo è costruito tramite elenchi seriali di associazioni sintagmatiche binarie. A livello microstrutturale, si riflette l'organizzazione bipartita del reale rinvenuta come basilare elemento caratterizzante nelle varie stratificazioni della società ed esemplificata nelle macrostrutture - ad indirizzo narrativo ed ideologicamente organizzato - di Autocoscienza di un servo, altro componimento della raccolta Week-end.

Se in quest'ultimo si tratta del binomio opposizionale padroneservo - con conseguenti correlati di morte e vita ed osmosi dei medesimi nella mancata assolutizzazione positiva o negativa dei due poli dell'antitesi - in questo testo la dicotomia si restringe al campo più genericamente esistenziale di morte-vita, con un analogo sommovimento dei confini distintivi, affrontati, intrecciati e sovrapposti in un'indagine profondamente terapeutica e stimolante.

Indicativa è la relazione isotopica che collega la suddetta dicotomia tematica alla struttura formale del testo: esso si articola in un filone binario di coppie lessematiche. Se all'interno di ogni coppia la sbarra interviene a suggerire graficamente la presenza del limite, dell'ostacolo, del confine da superare nella cercata integrazione dei due concetti centrali di vita-morte, lo stesso accoppiamento per divisione viene ribaltato su un'analoga colonna parallela di combinazioni sintagmatiche, ribadendo ulteriormente, nella duplicazione speculare della catena associativa, la struttura dualistica del tema. Conseguenza immediata di questo procedimento è lo scompiglio dell'univocità direzionale della lettura. In altri termini, diventano possibili sia una lettura orizzontale (che scavalca la distanza dalle coppie ricostituendole in un insieme continuo) che una verticale, dove le due colonne di coppie vengono lette separatamente, seguendo l'ipotesi distanziante dello spazio bianco intermedio.

La costruzione grafica del testo riflette adeguatamente lo sconvolgimento gnoseologico messo in atto dall'indagine poetica. Il fatto che i concetti opposizionali di vita-morte non compaiano in scontri univoci e differenzianti, ma si mescolino in una combinatoria deliberatamente ambigua, è comprovato - a livello tematico - dalle discordanti strutture interne delle coppie, i cui elementi sono legati alternatamente da rapporti di congiunzione-opposizione. Basti considerare rispettivamente alcuni esempi:

$\begin{array}{ll}\text { Opposizione } & \text { vita/morte } \\ & \text { fuori/dentro } \\ & \text { l'altro/io } \\ & \text { tu/io } \\ & \text { io/tutto } \\ \text { tutto/tu }\end{array}$


Congiunzione difese/vita acqua/vita spade/vita lotte/vita romba/vita buca/morte buca/vuoto

L'impossibilità di criteri univoci nella decifrazione del reale è ulteriormente sottolineata dall'accoppiamento di uno stesso termine, nell'ambito di varie coppie, ad elementi diversi tra di loro. Da un lato, infatti, l'associazione di due unità lessicali ad un medesimo termine permette di stabilire e perseguire affinità fra esse intercorrenti: in "tomba/uccello" il secondo termine ribalta sulla morte - e sul suo recettacolo sepolcrale - le prerogative del volo, alimentando cosí una catena sequenziale di questo tipo:

\section{tomba/uccello \\ vola/morte \\ buca/morte \\ buca/vola}

Analogamente, l'associazione di "uomo" a portavoci del mondo animale, vegetale o minerale tipo "cavallo," "uccello," "albero," "roccia," è sorretta dalla rispettiva combinazione di questi ultimi elementi e del termine umano di paragone con il lessema "tomba" (o il sostituto sinonimico "morte"):
tomba/uccello
uomo/uccello
cavallo/tomba
uomo/cavallo
tomba/albero
uomo/foglia
roccia/tomba
roccia/uomo

uomo/morte

Dall'altro lato, però, questi rapporti associativi intervengono a confondere le rispettive aree semantiche - e implicazioni esistenziali - di "morte" e "vita," che si trovano a condividere numerosi accoppiamenti sintagmatici e relative conseguenze:

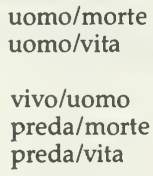

uomo/morte (osmosi rilevata da posizione parallela in lettura orizzontale) 
amo/morte (da considerare unitamente all'associazione sintagmatica semanticamente affine di "chiamo/tomba")

vita/amo

sudo/vita

ardo/morte ("sudo" e "ardo" accomunati dal sema "calore")

stringo/tomba tengo/vita (posizione parallela in lettura orizzontale)

$\mathrm{romba/vita}$

suono/tomba ("romba" e "suono" accomunati dal sema "rumore")

Le affinità delineate sovvertono ambiguamente lo schematico rigorismo del binomio opposizionale vita/morte. Ma la tentata esorcizzazione di quest'ultima tramite il suo parziale ribaltamento nell'area semantica della vita si conclude in un'impresa strozzata, irrisolta: le ultime due coppie lessematiche in ciascuna colonna ripropongono significativamente le immagini del vuoto e dell'assenza nei confronti della morte, qualificando indirettamente come nullo il tentativo di afferrarne l'essenza:

stringo/tomba

buca/vuoto tengo/vita

tomba/senza

E la mancata sintesi o integrazione dei due poli vita-morte in una visione conoscitiva soddisfacente si appoggia sul ribadimento dell'antitesi come struttura irriducibile del reale e dell'indagine ad esso collegata, a partire dai primi versi ("vita/morte") a quelli centrali (dove l'antitesi si sposta sul "fuori/dentro," "tu/io") alle già citate coppie finali. L'ineliminabile oppressione del limite si accampa ostinatamente nei punti strategici del testo, senza infiacchirne, però, la dinamica, inquisitiva espansione.

Nonostante il mancato approdo a certezze rassicuranti, il testo svolge un'indubbia funzione terapeutica in quanto anticipa e affronta la morte come elemento integrante dell'essere. È vero che la morte contrassegna la cessazione dei limiti del Dasein e la sua conversione nel tutto, nella completezza del "cerchio/morte," nella coincidenza del "fuori/dentro," dell" "io/tutto," ma appunto perché è trapasso al non-Dasein essa non è sperimentabile nel suo effettivo verificarsi, ma solo nel progressivo avvicinarsi ad essa, nell'angoscia vigile di un essere-per-la-morte, che nelle tentate esplorazioni della propria fine ribadisce, sí, la chiusura entro i limiti della propria finitezza, ma afferma al tempo stesso la propria libertà facendo della morte non l'oggetto di un incubo, ma una compagna al tempo stesso elusiva e assidua di vita. 
"(Visita alla necropoli di Norchia)" testimonia appunto l'infiltrazione diffusa e penetrante della morte nel campo di percezione dell'essere: e se la combinatoria sintagmatica che indaga sul binomio vita/morte ne scompiglia le rispettive implicazioni senza pervenire ad un rapporto felicemente coordinativo o nettamente differenziante fra le due realtà, è anche vero che la morte viene reintegrata nella vita, se non come mistero conquistato o conquistabile, come lucida, sofferta, tenace proiezione coscienziale verso il trascendimento - continuamente negato in vita - del proprio essere finito, come presenza imminente, ineluttabile, che grava sulla terra, sulle sue articolazioni di vita a vari livelli, sulla resistenza della materia inanimata, sull'isolato affiorare di tratti anatomici (lingua, occhi) e precipitazioni atmosferiche, sull'ubiquità testuale dell'uomo che si ripropone costantemente da un estremo all'altro della catena associativa alimentando questa tensione di congiungimento della vita e della sua cessazione. ${ }^{30}$

\section{Campione $n .3$}

Le calze infila, nere, e sfila, con i denti,

la spaccata, il doppio salto, in un istante, la calzamaglia,

all'indietro, capriola, poi la spaccata, i seni

premono il pavimento, dietro i capelli, dietro la porta,

non c'è, c'è il salto all'indietro, le cuciture,

l'impronta della mano, all'indietro, sul soffitto,

la ruota, delle gambe e delle braccia, di fianco,

dei seni, gli occhi, bianchi, contro il soffitto,

dietro la porta, calze di seta appese, la capriola.

Perché la tenda scuote, si è alzato,

il vento, nello spiraglio la luce, il buio,

dietro la tenda c'è, la notte, il giorno,

nei canali le barche, in gruppo, i quieti canali,

navigano, cariche di sabbia, sotto i ponti,

è mattina, il ferro dei passi, remi e motori,

i passi sulla sabbia, il vento sulla sabbia,

le tende sollevano $\mathrm{i}$ lembi, perché è notte,

giorno di vento, di pioggia sul mare,

dietro la porta il mare, la tenda si riempie di sabbia,

di calze, di pioggia, appese, sporche di sangue. ${ }^{31}$

In questo componimento, Porta evita le involuzioni del monologo interiore tramite l'adesione all'evento esterno, colto nel ritmo veloce e incalzante, asindetico, reiterativo (basti notare la frequente comparsa della preposizione "dietro"). Tutto suggerisce, foneticamente e semanticamente, il tentativo di abbattere un ostacolo: lessemi come "porta, tenda, pavimento, soffitto," e "vetro, 
scoglio, cemento, muro," che figurano abbondantemente nella raccolta da cui citiamo, si presentano come segni psicolinguistici di una realtà occultata che si vuole penetrare. Anche verbi come "premere, raschiare, affondare, flettersi, sfilare," e sostantivi come "spaccata, spiraglio, foro, taglio, lama" sono muniti di una carica aggressiva nei confronti del reale da scoprire, che si trincera dietro persistenti schermi protettivi. L'intenzionalità conoscitiva del gesto poetico, comunque, rimane tensione irrisolta in quanto manca íl finale superamento dell'ostacolo. Una continua progressione di dati antitetici, che spesso si raggruppano nello scontro violento di ossimori, ci rimanda nell'intero poemetto a questa situazione in bilico tra la volontà di ricerca e l'irraggiungibilità di certezze definitive: "Le calze infila, nere, e sfila, con i denti"; "non

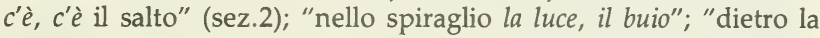
tenda c'è la notte, il giorno"; "perché è notte, giorno di vento" (sez.3). Constatiamo, qui, una realizzazione pratica dello "sviluppo per contraddizione" di cui parla Porta in Correlativo oggettivo, ${ }^{32}$ come segno fondamentale della sua poesia. Il segno non perde la propria naturale referenzialità, e nemmeno assistiamo ad accoppiamenti di segni irrelati, che potrebbero sottrarci alla possibilità di un racconto; è, invece, la continua tensione antitetica, la calcolata dialettica dei contrari, che impedisce una narrazione lineare, recuperando la funzionalità traumatica del linguaggio.

Siamo pertanto condotti a constatare che il testo è programmato come operazione metalinguistica: le reiterazioni sintattiche, foniche e semantiche instaurano insistentemente un rimando a se stesse, come dire, la chiusura del reale è innanzitutto chiusura del linguaggio. Tensione verbale e tensione come volontà di penetrazione del reale coincidono nel loro continuo rinvio speculare.

In definitiva, le tappe dell'iter poetico qui esaminate ci permettono di rilevare il serio compito contestatario di Porta, ossia la sua capacità di effettuare, tramite una sperimentazione eversiva nei confronti delle impalcature linguistico-poetiche tradizionali, continue revisioni dei canoni conoscitivi del reale, refrattarie a soste definitive e pericolose.

\section{York University}

\section{NOTE}

* Il presente saggio rappresenta una versione aggiornata ed ampiamente riveduta di un intervento preparato per l'annuale incontro della Canadian Society for Italian Studies tenutosi alla Laval University nel maggio 1976.

1 A. Giuliani, Introduzione, in I Novissimi. Poesie per gli anni '60 (Torino: Einaudi, 1965), p. 17. L'antologia è stata pubblicata per la prima volta nel 1961 da Rus- 
coni e Paolazzi, Milano. Comprende poesie di Elio Pagliarani, Alfredo Giuliani, Edoardo Sanguineti, Nanni Balestrini, Antonio Porta; ed à corredata da introduzione e note di A. Giuliani.

2 N. Balestrini, Linguaggio e opposizione, in I Novissimi, cit., pp. 196-97.

3 A Giuliani, Prefazione 1965, in I Novissimi, cit., p. 3, p. 5.

4 Ibid., p. 9.

5 E. Sanguineti, "Arte e morale. Un dibattito sull'estetica di Tolstoj," Marcatrè, 11-12-13 (1965), 31.

$6 \mathrm{Cfr}$. M. Corti, Il viaggio testuale. Le ideologie e le strutture semiotiche (Torino: Einaudi, 1978), p. 117.

7 A. Giuliani, Introduzione, in I Novissimi, cit., pp. 18-19.

8 A tale proposito Nanni Balestrini scrive: "È in un'epoca tanto inedita, imprevedibile e contradditoria, che la poesia dovrà più che mai essere vigile e profonda, dimessa e in movimento. Non dovrà tentare di imprigionare, ma di seguire le cose, dovrà evitare di fossilizzarsi nei dogmi, ed essere invece ambigua e assurda, aperta a una pluralità di significati e aliena dalle conclusioni per rivelare mediante un'estrema aderenza l'inafferrabile e il mutevole della vita" (Linguaggio e opposizione, in I Novissimi, cit., pp. 197-98).

9 U. Eco, Opera aperta (Milano: Bompiani, 1962), p. 267. In questo libro, Eco sostenendo la necessità di un'arte che assuma "un linguaggio in crisi per cogliere attraverso di esso la crisi di determinati rapporti" concernenti la situazione dell'uomo contemporaneo (p. 283) offre una precisa difesa estetića ed ideologica di tecniche linguistiche e poetica dei Novissimi.

10 A. Giuliani, Introduzione, in I Novissimi, cit., p. 16.

11 E. Sanguineti, Laborintus, (Varese: Magenta, 1956); ora in Catamerone 1951-1971, (Milano: Feltrinelli, 1974), p. 26.

12 Ibid., p. 45.

13 E. Sanguineti, Poesia informale? in I Novissimi, cit., p. 202.

14 A. Porta, "Di fronte alla luna" in La palpebra rovesciata (Milano: Azimuth, 1960), ora in $i$ rapporti, poesie 1958-1964 (Milano: Feltrinelli, 1966), p. 41.

15 N. Balestrini, Linguaggio e opposizione in I Novissimi, cit., p. 197.

$16 \mathrm{M}$. Corti, Il viaggio testuale, cit., p. 125.

17 F. Curi, "Poetica del nuovo terrore," Il Verri (marzo, 1970), 107.

18 A. Porta, "Correlativo oggettivo," Malebolge, 2 (1964), 69.

19 Ibid., 69.

20 E. Sanguineti, Ideologia e linguaggio (Milano: Feltrinelli, 1965). Postulando l'inscindibilità di ideologia e linguaggio l'autore afferma: "Non esiste originalità di visione ideologica, e di prospettiva realistica, che possa essere garantita da altro che dal linguaggio, giacchè la realtà di una opera, evidentemente e immediatamente, è una realtà linguistica. ... In un testo non vi è ideologia, esteticamente parlando, se non nella forma del linguaggio. . . . la polemica contro il naturalismo non sta già nell'usare un linguaggio stravolto rispetto a un linguaggio normale, ma nel tener conto che il linguaggio è sempre uno stravolgimento, cioè un modo di interpretare la realtà: un'ideologia": Gruppo 63. La nuova Letteratura. a cura di N. Balestrinie, A. Giuliani, (Milano: Feltrinelli, 1964), p. 382.

21 A. Porta, "Correlativo oggettivo," cit., 69.

22 A. Porta, Poesia e poetica, in I Novissimi, cit., p. 195.

23 A. Porta, dibattito in Gruppo 63. Il romanzo sperimentale, Palermo 1965 (Milano: Feltrinelli, 1965), p. 137.

24 A. Porta, Poesia e poetica in I Novissimi, cit., p. 194.

25 In uno scritto dal titolo "ll grado zero della poesia," Marcatrè (gennaio, 1964), Porta si richiama esplicitamente al senso del tragico di Karl Jaspers: "Il senso del tragico è alla base di ogni mia possibilità di operazione poetica" (p. 42), scrive tra l'altro il poeta; il processo dell'esistenza non è un integrarsi ordinato di elementi dialettici, ma un succedersi e coesistere di irriducibili antinomie. "That reality is split, that truth is divided — ha scritto Jaspers — "is a basic insight of tragic knowledge": Tragedy is not enough, trad. di H.A.T. Reiche, H.T. Moore (New York: Archon Books, 1969), p. 57. Un'affermazione di Enzo Paci a 
proposito del filosofo tedesco risulta infatti applicabile allo stesso Porta: "la verità è dunque irraggiungibile: è un orizzonte che si sposta sempre al di là del piano nel quale si pretende di afferrarlo, un orizzonte circoscrivente che non può essere circoscritto (Umgreifende)": E. Paci, La filosofia contemporanea (Milano: Garzanti, 1957), p. 229.

26 A. Porta, Metropolis (Milano: Feltrinelli, 1971), pp. 11-12.

$27 \mathrm{Ci}$ siamo rifatti a Marcuse in quanto nel capitolo "The closing of the universe of discourse," in One-dimensional man (Boston: Beacon Press, 1964), pp. 84-120, egli si occupa del problema del linguaggio contemporaneo. Anche secondo Marcuse il linguaggio chiuso, repressivo ed autoritario si è imposto nella presente civiltà. "The closed language" - scrive il filosofo nell'opera sopra menzionata - "does not demonstrate and explain - it commands decision, dictum, command. . . . This language controls by reducing the linguistic forms and symbols of reflections, abstraction, development, contradiction, by substituting images for concepts. It denies or absorbs the trascendent vocabulary, it does not search for but establishes and imposes truth and falsehood." (p. 101, p. 103). Il nostro accostamento al pensiero di Marcuse è inoltre sostenuto da una recensione dello stesso Porta a L'uomo a una dimensione nella quale anticipando certe riflessioni che poi verranno sviluppate in sede poetica proprio in Metropolis, tra l'altro scrive: "Voglio dire che si patiscono insulti quotidiani, il peggiore dei quali rimane il falso nell'informazione, l'informazione sottratta": Quindici, n. 1 (1967), p. 2. Non riteniamo opportuno, ai fini del nostro discorso, approfondire taluni legami tra la poesia di Porta o dei Novissimi in genere e il pensiero degli esponenti della Scuola di Francoforte. Ci limitiamo a ricordare che essi, come i Novissimi, concepiscono l'arte solo nella sua funzione di negazione nei confronti dei "valori" imposti dalla società neocapitalistica. La demolizione o la negazione di tali "valori" si realizza innanzitutto tramite l'assunzione di un "linguaggio della negazione." Solo l'arte d'avanguardia, come scriverà Marcuse, presenta "lo sforzo di superare il potere dei fatti sul mondo, di parlare un linguaggio che non sia il linguaggio di coloro che stabiliscono i fatti, impongono l'obbedienza a essi e ne traggono profitto." La dialettica e il linguaggio poetico, piuttosto, si trovano sullo stesso piano. L'elemento comune consiste nella ricerca di un "linguaggio autentico," il linguaggio della negazione come il Grande Rifiuto di accettare le regole di gioco in cui i dati sono falsati: $\mathrm{H}$. Marcuse, Ragione e rivoluzione (Bologna: Il Mulino, 1966), pp. 15-16.

$28 \mathrm{La}$ lontana matrice di quest'operazione potrebbe essere il Dictionnaire des idées reçues di Flaubert. A differenza di Porta comunque, il romanziere francese si limita a registrare con la più neutra impassibilità le idee, i luoghi comuni ed $\mathrm{i}$ detti proverbiali coniati dalla sua epoca. Flaubert diventa l'artista-spettatore spassionato, immune da ogni demistificazione o illusione giudicante. L'operazione demistificatoria di montaggio e di neutralizzazione semantica proposta da Porta si colloca dunque in un'area nettamente antitetica a quella di Flaubert.

29 A. Porta, "(Visita alla necropoli di Norchia)," in Week-end (Roma: Cooperativa Scrittori, 1974), p. 28. L'analisi di questo componimento è stata incorporata con qualche lieve variante nel mio scritto "Linguaggio come terapia nell'ultimo Porta," Strumenti critici, 39-40 (1979), 349-62.

30 Il nucleo fenomenologico-esistenziale che abbiamo rintracciato in questo componimento di Porta (la "decisione anticipatrice" della morte collegata alla tensione del poter-essere-un-tutto, ossia alla ricerca dell'autenticità) si riallaccia chiaramente - a nostro avviso - alla filosofia heideggeriana. "Poiché l'anticipazione della possibilità insuperabile, - ha scritto Heidegger - apre, nel contempo, alla comprensione delle possibilità situate al di qua di essa, essa porta con sé la possibilità dell'anticipazione esistentiva dell'esserci totale, cioè la possibilità di esistere concretamente come poter-essere totale": Essere e tempo, trad. di P. Chiodi (Torino: UTET, 1969), p. 396. Anche G. Pontiggia, nella sua prefazione alla raccolta di Porta Quanto ho da dirvi (Milano: Feltrinelli, 1977), pp. 7-13, riconduce alcune costanti della poesia portiana alla filosofia di Heidegger, evidenziando nell'opera del poeta la connessione tra "vedere, conoscere e angoscia." 
31 A. Porta, Aprire (Milano: Scheiwiller, 1964), sez. 2-3, pp. 15-16.

32 A. Porta, Correlativo oggettivo, cit., 69. 